\title{
Sound field separation for planar sources facing a parallel reflector
}

\author{
Elias Zea ${ }^{a, *}$ Ines Lopez Arteaga ${ }^{a, b}$ \\ ${ }^{a}$ KTH Royal Institute of Technology, The Marcus Wallenberg Laboratory for Sound and Vibration Research, Teknikringen 8, \\ SE-100 44 Stockholm, Sweden \\ ${ }^{b}$ Eindhoven University of Technology, Department of Mechanical Engineering, 5600 MB Eindhoven, The Netherlands
}

\begin{abstract}
We consider the problem of separating and reconstructing the sound field radiated by a planar source, e.g. a vibrating plate, that faces a parallel reflector. For this purpose we propose a Fourier-based technique to process near-field microphone array measurements that are performed on a single plane between the plate and the reflector. A closed-form expression is derived in the wavenumber domain to recover the free-space sound field radiated by the plate. The fundamental assumption is that the plate scatters sound in a locallyreacting fashion. Overall, provided the admittances of the plate and the reflecting surface are known, the results demonstrate that it is possible to recover the free-field sound radiated by the plate with a reasonable accuracy.
\end{abstract}

Keywords: sound field separation, NAH, planar sources, reverberant environments

\section{Introduction}

For a few decades microphone arrays have been used to characterize and visualize the sound field radiated by vibro-acoustic sources. One of the pioneering techniques is near-field acoustic holography (NAH), developed in the ' 80 s by Williams and collaborators $[1,2]$. Considering a planar array geometry, the NAH formulation imposes the assumption that all sources are confined to one of the sides of the array, and that the opposite side is source- and reflection-free, namely silent. However, this requirement cannot always be met because of the acoustic environment in which the measurement is performed. For example, the presence of reflecting surfaces or additional sources in the vicinity of the array introduces disturbances in the measured sound and causes the sound sources to be inaccurately characterized.

To this end two approaches are typically adopted: (i) the measurement is performed in an echo-free chamber, or (ii) the pressure (or particle velocity) is measured in two parallel planes or layers. The former approach requires a conventional single layer array but involves moving the source from its original location, which in certain situations may be too costly and troublesome. The alternative approach requires a double layer array, i.e. twice as many sensors, yet it allows the measurements to be performed in-situ, that is, it circumvents the need of moving the source.

The idea of double layer arrays, first introduced in the '50s by Pachner [3] and followed later by Weinreich and Arnold [4], has given rise to the present-day concept of sound field separation techniques (FST). The fundamental principle of FST is to remove the sound due to reflections and scattering, as well as due to the presence of disturbing sources other than the one of interest. FST have either a space-domain formulation or a wavenumber-domain formulation. Within these two categories, typical array topologies are two layers of pressure or particle velocity transducers, or one layer of pressure-velocity (so-called p-u) probes. (For the sake of brevity we shall here limit the literature review to some applications of FST involving planar array

\footnotetext{
*Corresponding author. Tel. +4687907903.

Email addresses: zea@kth.se (Elias Zea), inesla@kth.se (Ines Lopez Arteaga)

$U R L:$ https://www.researchgate.net/profile/Elias_Zea (Elias Zea)
} 
geometries, and the interested reader is referred to Section 1 in [5] for a more comprehensive summary of the state-of-the-art FST.) Acknowledged examples of space-domain formulations are based on the equivalent source method (ESM) [6, 7, 8, 9, 10, 11], and the statistically optimized NAH method $[12,13,14,15,16,17]$. On the other hand, wavenumber-domain (or Fourier-based) methods have found abundant application not only in the context of NAH [18, 19, 20, 21], but also for the determination of the plane-wave reflection coefficient of absorbing surfaces [22, 23, 24]. In particular, the method developed by Bi et al. [11] would the most applicable to the scenario examined in this paper; nonetheless there is at present no single layer (and wavenumber-domain) method with an equivalent potential.

As opposed to double layer methods, the technique introduced by the authors in [5] aims at processing single layer microphone array measurements of compact sources in the presence of a reflector that is parallel to the array. This technique, here referred to as the first-order reflection (FOR) method, is based on a wavenumber-domain formulation of a popular model in the seismic signal processing community: WRW (W denotes wave propagation and $\mathrm{R}$ denotes reflection) $[25,26]$. It is assumed in the FOR method that the reflector is locally-reacting, hence the pressure (particle velocity) at every point depends only on the pressure (particle velocity) at that point [27]. Moreover, as mentioned in [5], a model for extended planar sources can potentially be formulated by making an analogy with the WRW model for two reflectors [26].

The acoustic problem of interest in this work is that of a planar source, such as a vibrating plate, that faces a reflecting surface. This particular acoustic scenario is intrinsically new, as there are no experimental works in the literature to the authors' knowledge. A possible application example of such a situation is the region underneath a vehicle facing asphalt, provided the smallest wavelength to be recovered is much larger than the surface irregularities of the vehicle underbody. Unlike the case of a compact source and the FOR method, there are in theory infinitely many reflected and scattered waves between the plate and the reflector. The key novelty of this research is then the introduction and experimental study of a wavenumberdomain method, whose aim is to process single layer microphone array measurements performed between the plate and the reflector. The main assumption is that the vibrating plate scatters sound as a locally-reacting surface, and the central requirement is the knowledge of the admittance of the plate as well as of the reflector (e.g. via Tamura's method [23]). The overall results demonstrate that the introduced method is capable of separating and reconstructing the free-field sound radiation of the plate within a reasonable accuracy.

\section{Theory}

Let us first decompose the sound field measured at the microphone array into two main components: direct field $\mathbf{p}_{h, \text { ff }}$ and reverberant field $\mathbf{p}_{h, \text { rv }}$. The former is equivalent to the sound field radiated by the plate in an anechoic chamber, whereas the latter is the sound field due to the presence of the parallel reflector as well as the scattering due to the spatial extent of the plate. The following sections present each of these definitions, and ends with the inverse problem formulation as well as the required regularization. Throughout the paper the time-harmonic dependence of the sound fields is omitted.

\subsection{Direct field and first-order reflection}

As illustrated in Fig. 1, the hologram or measurement plane is located in the plane $z=z_{h}$, and the plate is located in the plane $z=z_{p}<z_{h}$. In Fig. 1(a), the direct sound field consists of the acoustic waves propagated from the plate plane $z_{p}$ to the hologram plane $z_{h}$. Then, as shown in Fig. 1(b), the first-order reflected field comprises the propagation from $z_{h}$ to $z_{1}$, the acoustic reflection at the surface $z_{1}$, and propagation from $z_{1}$ to $z_{h}$. This field shall be denoted as $\mathbf{p}_{\text {ref,1, }}$, and it is related to the direct field via the WRW model as follows

$$
\mathbf{p}_{\mathrm{ref}, 1}=\mathbf{W}_{1 h} \mathbf{R}_{1} \mathbf{W}_{h 1} \mathbf{p}_{h, \mathrm{ff}},
$$

where the matrix $\mathbf{W}_{m n}$ contains the Dirichlet Green's functions characterizing the wave-field extrapolation from plane $z_{m}$ to plane $z_{n}$, whilst the matrix $\mathbf{R}_{1}$ contains the reflectivity impulse responses of the surface $z_{1}[25,26]$. See Appendix A for further details on the structure of these matrices. 
(a)

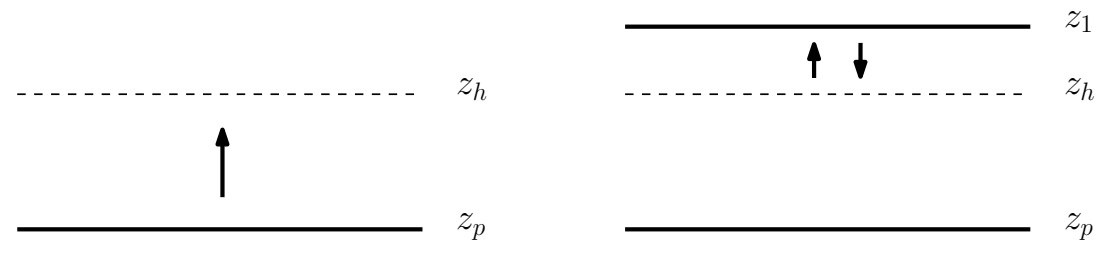

Figure 1: Geometrical illustration of (a) the direct field and (b) the first-order reflected field, where $z_{p}$ is the plate plane, $z_{h}$ is the hologram (measurement) plane, and $z_{1}$ is the reflecting plane.

Given the above geometry as well as a spatially homogeneous reflecting surface, the convolution theorem holds $[28,29]$, and the above equation can be written in the wavenumber domain by means of spatial Fourier transforms as

$$
\tilde{\mathbf{p}}_{\mathrm{ref}, 1}=\tilde{\mathbf{R}}_{1} \mathbf{G}_{h 1}^{2} \tilde{\mathbf{p}}_{h, \mathrm{ff}}=\mathbf{G}_{\mathrm{ref}} \tilde{\mathbf{p}}_{h, \mathrm{ff}},
$$

with $\mathbf{G}_{m n}=\operatorname{diag}\left(\mathrm{e}^{\mathrm{j} k_{z}\left(z_{n}-z_{m}\right)}\right)$ for planes $z_{m}$ and $z_{n}, \mathrm{j}^{2}=-1$, and the wavenumber $k_{z}$ being real for propagating waves and imaginary for evanescent waves

$$
k_{z}= \begin{cases}\sqrt{k^{2}-k_{x}^{2}-k_{y}^{2}}, & k^{2} \geq k_{x}^{2}+k_{y}^{2}, \\ \mathrm{j} \sqrt{k_{x}^{2}+k_{y}^{2}-k^{2}}, & k^{2}<k_{x}^{2}+k_{y}^{2}\end{cases}
$$

Here $k \in \mathbb{R}^{+}$is the acoustic wavenumber and $\left(k_{x}, k_{y}\right)$ are spatial vectors along the $x$ and $y$ directions.

In addition, the matrix operator $\tilde{\mathbf{R}}_{i}$ contains the plane-wave reflection coefficient [27, 30] of the surface $z=z_{i}$, that is ${ }^{1}$

$$
\tilde{\mathbf{R}}_{i}=\operatorname{diag}\left(\frac{\cos \theta-\beta_{i}}{\cos \theta+\beta_{i}}\right),
$$

where $\theta=\cos ^{-1}\left(k_{z} / k\right)$ is the plane-wave incidence angle, and $\beta_{i} \in \mathbb{C}$ is the specific acoustic admittance at the plane $z_{i}$, in this case the reflecting plane $z_{1}$. Underlying this expression resides the assumption of the reflector being locally-reacting, which means that the pressure (particle velocity) at every point on the surface only depends on the pressure (particle velocity) at that point [27]. Moreover, the reflector size is considered to be large with respect to the acoustic wavelength $\lambda=2 \pi / k$.

\subsection{Scattered field and higher-order reflected fields}

If the plate dimensions are large with respect to the acoustic wavelength, the first-order reflected field $\mathbf{p}_{\text {ref,1 }}$ will likely be back-scattered by the plate surface (see Fig. 2). It is then further assumed that the vibrating plate has the properties of a locally-reacting surface like the reflector $z_{1}$. Hence the scattered sound field comprises the propagation of the first-order reflected field towards the plate $z_{p}$, the locally-reacting reflection from the plate, and the propagation towards the hologram plane $z_{h}$. In mathematical terms,

$$
\tilde{\mathbf{p}}_{\mathrm{sca}, 1}=\tilde{\mathbf{R}}_{p} \mathbf{G}_{p h}^{2} \tilde{\mathbf{p}}_{\mathrm{ref}, 1}=\mathbf{G}_{\mathrm{sca}} \tilde{\mathbf{p}}_{\mathrm{ref}, 1}=\mathbf{G}_{\mathrm{sca}} \mathbf{G}_{\mathrm{ref}} \tilde{\mathbf{p}}_{h, \mathrm{ff}} .
$$

The next order of reflection from the surface $z_{1}$ is similarly defined as in Eq. (2), but replacing the direct sound field by the scattered sound field $\tilde{\mathbf{p}}_{\text {sca }, 1}$. Thus it follows that

$$
\tilde{\mathbf{p}}_{\mathrm{ref}, 2}=\mathbf{G}_{\mathrm{ref}} \tilde{\mathbf{p}}_{\mathrm{sca}, 1}=\mathbf{G}_{\mathrm{sca}} \mathbf{G}_{\mathrm{ref}}^{2} \tilde{\mathbf{p}}_{h, \mathrm{ff}} .
$$

\footnotetext{
${ }^{1}$ The subscript notation $i$ ought not to be confused with the $i$-th element of the matrix.
} 


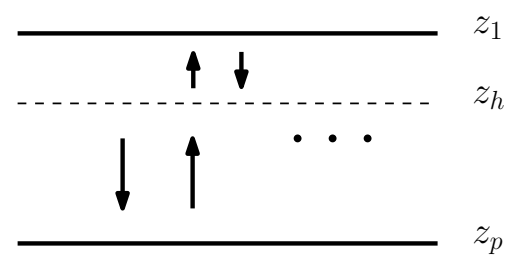

Figure 2: Geometrical illustration of the scattered field from the plate plane $z_{p}$, as well as higher-order reflected fields.

Thereafter, the summation of infinite orders of reflection and scattering, both due to the reflector and the plate, gives a Neumann series of the form:

$$
\tilde{\mathbf{p}}_{h, \mathrm{rv}}=\sum_{q=1}^{\infty}\left(\tilde{\mathbf{p}}_{\mathrm{ref}, q}+\tilde{\mathbf{p}}_{\mathrm{sca}, q}\right)=\sum_{q=1}^{\infty}\left[\mathbf{G}_{\mathrm{sca}}^{q-1} \mathbf{G}_{\mathrm{ref}}^{q}+\left(\mathbf{G}_{\mathrm{sca}} \mathbf{G}_{\mathrm{ref}}\right)^{q}\right] \tilde{\mathbf{p}}_{h, \mathrm{ff}},
$$

which converges to the expression

$$
\tilde{\mathbf{p}}_{h, \mathrm{rv}}=\left(\mathbf{G}_{\mathrm{ref}}+\mathbf{G}_{\mathrm{sca}} \mathbf{G}_{\mathrm{ref}}\right)\left(\mathbf{I}-\mathbf{G}_{\mathrm{sca}} \mathbf{G}_{\mathrm{ref}}\right)^{-1} \tilde{\mathbf{p}}_{h, \mathrm{ff}},
$$

where $\mathbf{I}$ is the identity matrix. Lastly, the hologram pressure field is constructed as a function of the free-field sound pressure as

$$
\tilde{\mathbf{p}}_{h}=\tilde{\mathbf{p}}_{h, \mathrm{ff}}+\tilde{\mathbf{p}}_{h, \mathrm{rv}}=\mathbf{G} \tilde{\mathbf{p}}_{h, \mathrm{ff}},
$$

with

$$
\mathbf{G}=\mathbf{I}+\tilde{\mathbf{R}}_{1}\left(\mathbf{I}-\tilde{\mathbf{R}}_{p} \tilde{\mathbf{R}}_{1} \mathbf{G}_{p 1}^{2}\right)^{-1}\left(\mathbf{G}_{h 1}^{2}+\tilde{\mathbf{R}}_{p} \mathbf{G}_{p 1}^{2}\right)
$$

It is demonstrated in Appendix B that this derivation is equivalent to a wavenumber-domain formulation of the WRW model for two reflecting surfaces [26], assuming that the vibrating plate is one of such surfaces.

\subsection{Regularized inversion}

The inverse problem entails the recovery of the free-field pressure, $\tilde{\mathbf{p}}_{h, \mathrm{ff}}$, from measurements $\tilde{\mathbf{p}}_{h}$ contaminated with reflections as well as background noise. In other words, it comprises the inversion of the operator G in Eq. (10), for which a regularization technique is essential. As shown in Appendix C, this operator can potentially have zero singular values at the radiation circle $k^{2}=k_{x}^{2}+k_{y}^{2}$, although in practice this only happens when the measurement aperture follows $L=\lambda\left(m^{2}+n^{2}\right)^{1 / 2}$ for integers $(m, n)$.

In this paper we wish to make a comparison of the proposed methods with respect to the free-field NAH method [2] and the first-order reflection (FOR) model [5]; hence the sound field will further be reconstructed at a plane $z=z_{s}$ closer to the vibrating plate -i.e. $z_{p}<z_{s}<z_{h}$. The latter implies the substitution of the mathematical expression $\tilde{\mathbf{p}}_{h, \mathrm{ff}}=\mathbf{G}_{s h} \tilde{\mathbf{p}}_{s}$ in Eq. (9). To this end we shall define the operator $\overline{\mathbf{G}}=\mathbf{G}_{s h} \mathbf{G}$, such that the measured pressure $\tilde{\mathbf{p}}_{h}$ can be related to the reconstructed pressure $\tilde{\mathbf{p}}_{s}$.

Provided the aforementioned modifications and the choice of a modified Tikhonov regularization approach, the inverse solution follows from minimizing the optimization functional

$$
\mathcal{F}\left(\tilde{\mathbf{p}}_{s}\right)=\frac{1}{2}\left\|\tilde{\mathbf{p}}_{h}-\overline{\mathbf{G}} \tilde{\mathbf{p}}_{s}\right\|_{2}^{2}+\mu^{2} \frac{1}{2}\left\|\mathbf{L} \tilde{\mathbf{p}}_{s}\right\|_{2}^{2}
$$

where $\mu>0$ is the Tikhonov regularization parameter. The role of $\mathbf{L}$ is to penalize the reconstructed pressure spectrum at wavenumbers for which $\overline{\mathbf{G}}$ is close to zero, and its definition follows from the modified Tikhonov matrix [31]

$$
\mathbf{L}=\mu^{2}\left(\overline{\mathbf{G}}^{H} \overline{\mathbf{G}}+\mu^{2} \mathbf{I}\right)^{-1}
$$

where superscript $H$ denotes the Hermitian matrix transpose. Then, provided the reflector admittance $\beta_{1}$ and the plate admittance $\beta_{p}$ are known, the global minimum of the functional in Eq. (11) follows

$$
\tilde{\mathbf{p}}_{s}=\boldsymbol{\Gamma} \overline{\mathbf{G}}^{-1} \tilde{\mathbf{p}}_{h},
$$


where

$$
\boldsymbol{\Gamma}=\left(\overline{\mathbf{G}}^{H} \overline{\mathbf{G}}+\mu^{2} \mathbf{L}^{H} \mathbf{L}\right)^{-1} \overline{\mathbf{G}}^{H} \overline{\mathbf{G}}
$$

is the so-called modified Tikhonov regularization filter [31].

The regularized free-field NAH solution [31] is obtained by setting $\overline{\mathbf{G}}=\mathbf{G}_{s h}$ in the above equation; whereas the regularized solution to the FOR model [5] is found by equating $\overline{\mathbf{G}}=\mathbf{G}_{s h}+\mathbf{G}_{h 1} \tilde{\mathbf{R}}_{1} \mathbf{G}_{s 1}$. It should be noted that $\mathbf{L}$ must change accordingly for the free-field NAH and FOR regularized solutions.

\subsubsection{Choice of regularization parameter}

One more point that ought to be stressed concerns the choice of the regularization parameter $\mu$. In practice, this parameter is found by means of an iterative criterion that establishes a trade-off between the sharpness and the noise of the regularized solution [31, 32]. Among these criteria, some do not require knowledge of the hologram noise variance, such as the L-curve [33] and the generalized cross-validation [34], whereas others, such as Morozov's discrepancy principle [35], do require an estimate of the variance. In this paper we adopt the L-curve criterion, which entails the computation of the corner of the curve $\log \eta(\mu)$ versus $\log \rho(\mu)$, where

$$
\begin{gathered}
\eta(\mu)=\left\|\tilde{\mathbf{p}}_{s}\right\|_{2}=\left\|\boldsymbol{\Gamma} \overline{\mathbf{G}}^{-1} \tilde{\mathbf{p}}_{h}\right\|_{2} \\
\rho(\mu)=\left\|\overline{\mathbf{G}} \tilde{\mathbf{p}}_{s}-\tilde{\mathbf{p}}_{h}\right\|_{2}=\left\|(\mathbf{I}-\boldsymbol{\Gamma}) \tilde{\mathbf{p}}_{h}\right\|_{2} .
\end{gathered}
$$

The optimal $\mu$ used in Eq. (13) is then given by the curve's corner, and the latter is obtained with the "l_corner" routine of Regularization Tools MATLAB toolbox ${ }^{2}$.

\section{Experimental study}

\subsection{Setup}

A photograph of the experimental setup is shown in Fig. 3. The measurements are carried out in the anechoic chamber of the Marcus Wallenberg Laboratory for Sound and Vibration Research (KTH). A scanning measurement procedure is adopted with a $1 / 4^{\prime \prime}$ GRAS BH40 free-field microphone. The latter is

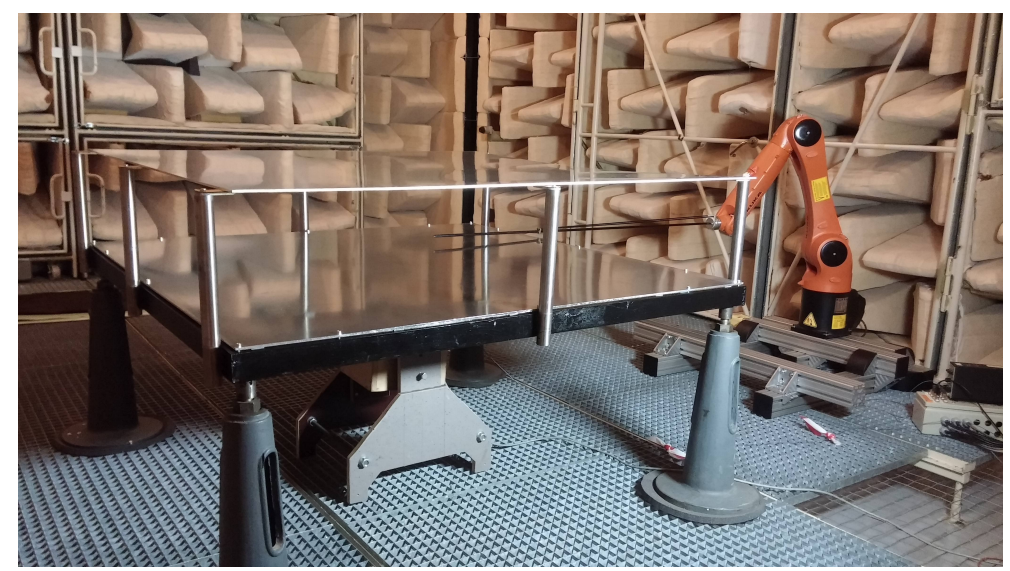

Figure 3: Photograph of the experimental setup.

mounted on a thin carbon fibre pipe, which is attached to the tool tip of a KUKA robot ${ }^{3}$. The pipe is 1.5 $\mathrm{m}$ long, with $10 \mathrm{~mm}$ outer diameter and $8 \mathrm{~mm}$ inner diameter, and its purpose is to minimize any potential scattering due to the robot ${ }^{4}$. The robot model is KR10 R1100 sixx WP, and the scanning routine is fully automated with a KR C4 controller and an NI-DAQx multi-channel acquisition system. The measurement

${ }^{2}$ See http://www2. compute.dtu.dk/ pcha/Regutools/ for more details. 
aperture is $0.6 \times 0.6 \mathrm{~m}^{2}$, with sampling steps of $\Delta=2 \mathrm{~cm}$ at $31 \times 31$ microphone positions. The temporal sampling frequency is $10 \mathrm{kHz}$.

The source consists of a $1.5 \times 1.5 \mathrm{~m}^{2}$ AW-6082 aluminium plate of $5 \mathrm{~mm}$ thickness, and it is screwed at 16 positions to a steel frame that lies on four rigid supports. In addition to the screws, there is a layer of isolating material between the plate and the frame, in order to minimize the plate velocity at the edges. The excitation mechanism is a shaker located at the center of the plate, and the excitation signal is white noise within a $4.5 \mathrm{kHz}$ bandwidth.

The reflecting surface consists of another $1.5 \times 1.5 \mathrm{~m}^{2}$ aluminium plate, with same thickness, and it lies on seven cylindrical steel supports that are screwed to the sides of the rigid frame described above. There is no cylindrical support on the side towards the robotic arm such that it can freely move, and there are $5 \mathrm{~mm}$ thick isolating pads between the cylinders and the reflector, so as to minimize potential structural vibrations coming from the plate and/or the frame. The distance between the plate and the reflector is $20.6 \mathrm{~cm}$. It shall be said that the vibrations of the reflector have been measured with accelerometers, and they are at least five orders of magnitude weaker than those of the vibrating plate; thus the reflector can be deemed passive and its sound radiation can be neglected.

The microphone measurements are performed in the presence and in the absence of the reflector. The measurements in the presence of the reflector are done at the hologram plane $z_{h}$, which is $8 \mathrm{~cm}$ above the vibrating plate, and correspond to the input $\mathbf{p}_{h}$ for all reconstruction methods. The measurements in the absence of the reflector are again performed at the hologram plane $z_{h}$, corresponding to $\mathbf{p}_{h}$,ff, and these are used to estimate the contribution of the reverberant field to the total sound field $\mathbf{p}_{h}$. The reference (ground-truth) pressure is measured with the reflector absent, at the reconstruction plane $z_{s}$, which is $3 \mathrm{~cm}$ above the vibrating plate.

\subsection{Results}

The sound field is reconstructed via the free-field (FF) NAH method [2], the first-order reflection (FOR) method [5], and the introduced method, here denoted as the locally-reacting plate (LRP) method. The frequency range considered is from $200 \mathrm{~Hz}$ to $2000 \mathrm{~Hz}$, in steps of $50 \mathrm{~Hz}$. In addition, 6-th order linear predictive border padding [36] is applied to $\mathbf{p}_{h}$ prior to the spatial Fourier transformation, in order to extrapolate the spatial aperture to $2.02 \times 2.02 \mathrm{~m}^{2}$ and minimize windowing artifacts ${ }^{5}$.

The accuracy of the reconstructions is evaluated with the error metric

$$
\varepsilon=20 \log _{10} \frac{\left\|\mathbf{p}_{s}^{\mathrm{ref}}-\mathbf{p}_{s}^{\mathrm{rec}}\right\|_{2}}{\left\|\mathbf{p}_{s}^{\mathrm{ref}}\right\|_{2}},
$$

where superscripts "rec" and "ref" denote reconstructed and reference pressure fields respectively.

\subsubsection{Minimum error approach}

The lack of knowledge of the admittances is a problem that makes the functional in Eq. (11) nonlinear and under-determined. We shall instead find what the best reconstructions are irrespective of the knowledge of the admittances $\beta_{1}$ and $\beta_{p}$. (Comments on the applicability of the method, specially concerning knowledge of the admittances, are given in Section 4.2 of this manuscript.) The basic idea is to make use of the knowledge of the reference pressure field, $\mathbf{p}_{s}^{\text {ref }}$, in order to compute the minimum reconstruction error provided a set of admittances. Thus, for instance, the so-called optimal reflector admittance in the FOR method [5] is obtained by solving

$$
\beta_{1}=\arg \min _{\bar{\beta}_{1}} \varepsilon\left(\bar{\beta}_{1}\right)
$$

\footnotetext{
${ }^{3}$ See https://www.kuka.com/en-us/products/robotics-systems/industrial-robots for further information.

${ }^{4}$ Considering a scattering bound $\lambda_{s c} \leq 10 D_{\text {out }}$, where $D_{\text {out }}$ is the outer pipe diameter, then scattering is likely to occur at $f \geq 3.43 \mathrm{kHz}$ in lossless air media.

${ }^{5}$ The extrapolated aperture gives a wavenumber spacing of $\Delta k \approx 3.1 \mathrm{rad} / \mathrm{m}$, thus at least one propagating wave can be resolved for frequencies above $200 \mathrm{~Hz}$.
} 
where $\bar{\beta}_{1}$ is a set of 225 values of the complex unit circle with positive real part (passive element).

Following a similar reasoning, the minimization for the LRP model depends on $\beta_{1}$ and $\beta_{p}$. In mathematical terms,

$$
\left(\beta_{1}, \beta_{p}\right)=\arg \min _{\bar{\beta}_{1}, \bar{\beta}_{p}} \varepsilon\left(\bar{\beta}_{1}, \bar{\beta}_{p}\right) .
$$

As opposed to the reflecting surface, the vibrating plate plays the role of an active element in the system, thus the set $\bar{\beta}_{p}$ spans 225 values of the complex unit circle with negative real part. Provided the complex admittances $\bar{\beta}_{1}$ and $\bar{\beta}_{p}$ are organized into vectors, the dimensionality of this minimization problem is two.

It ought to be stressed here that these optimal admittance values $\beta_{1}$ and $\beta_{p}$ need not be entirely physically meaningful, since the minimization includes modelling effects such as spatial extrapolation and regularization. Additionally, these admittance values might as well account for the steel frame and the cylindrical supports between the frame and the reflector. All the same, the reasoning behind the minimum error approach is that there does exist a solution space in which the admittances $\left(\beta_{1}, \beta_{p}\right)$ yield minimum reconstruction error, and that there is potential for accurate reconstructions if the admittances, or their estimates, are known (e.g. via Tamura's method [23]).

\subsubsection{Performance of free-field $N A H$}

As an initial analysis this section shows reconstruction results for the FF method. A complementary quantity we shall make use of is the reverberant-to-direct ratio (RDR), which is defined in $\mathrm{dB}$ as

$$
\mathrm{RDR}=20 \log _{10} \frac{\left\|\mathbf{p}_{h, \mathrm{rv}}\right\|_{2}}{\left\|\mathbf{p}_{h, \mathrm{ff}}\right\|_{2}},
$$

where $\mathbf{p}_{h, \mathrm{rv}}=\mathbf{p}_{h}-\mathbf{p}_{h, \mathrm{ff}}$ is an estimate of the contribution of the reverberant field to the total sound field. In few words, a positive (negative) RDR indicates that the reverberant field is stronger (weaker) than the direct field, and this in turn dictates the reconstruction accuracy of the FF method [2].

An illustration of the total, direct and reverberant fields is depicted in Fig. 4, in which the sound pressure level (SPL) in $\mathrm{dB}$ re $20 \mu \mathrm{Pa}$ is shown at three frequencies of interest: $350 \mathrm{~Hz}$ (top row), $800 \mathrm{~Hz}$ (middle row) and $1800 \mathrm{~Hz}$ (bottom row). The RDR values computed via Eq. (19) at these frequencies are $-6 \mathrm{~dB}$, $-2.9 \mathrm{~dB}$ and $2.7 \mathrm{~dB}$ respectively. By looking at Figs. 4(b) and (c), as well as Figs. 4(e) and (f), it can be qualitatively confirmed that the reverberant field is indeed weaker than the direct field. A similar (yet opposite) conclusion can be drawn in Figs. 4(h) and (i), where the SPL of the reverberant field seems to be comparable to and sometimes higher than that of the direct field. It can therefore be expected that the reconstruction accuracy via the $\mathrm{FF}$ method at $800 \mathrm{~Hz}$ and $1800 \mathrm{~Hz}$ is likely poorer than at $350 \mathrm{~Hz}$.

Fig. 5 shows the reconstruction error via the FF method and the RDR within the whole frequency range of interest. The reconstruction error closely follows the RDR and this confirms the reasoning presented above. It must however be mentioned that there is an implicit error due to background noise in the estimation of $\mathbf{p}_{h, \mathrm{rv}}$, which might influence how the reconstruction error actually follows the RDR. Moreover, the peaks in these curves can be also linked to the resonances due to the geometry of the problem: e.g. the maximum at around $830 \mathrm{~Hz}$ corresponds with half a wavelength $(\lambda / 2 \approx 20.6 \mathrm{~cm})$ between the plate and the reflector. Overall, the reconstruction errors via the FF method are on average between $0 \mathrm{~dB}$ and $-5 \mathrm{~dB}$.

\subsubsection{Performance of first-order reflection and locally-reacting plate models}

The reconstruction errors versus frequency for the FOR and LRP models are shown in Fig. 7. These errors are found by means of adopting the minimum error approach presented in Eqs. (17) and (18). For the sake of a more accurate comparison the reconstruction error via the FF method is also included. The errors obtained with the LRP model indicate an improvement of about (and sometimes larger than) $2 \mathrm{~dB}$ over the FOR model. However, at some frequencies, the improvement may arguably be too small to be considered beneficial; see e.g., Fig. 7 at $700 \mathrm{~Hz}$. Overall, as expected, the reconstruction errors in via both FOR and LRP models are lower than those via the FF method.

The reader might at this point question why the reconstruction errors shown here are higher than those typically obtained with, for example, NAH in free-field conditions - usually between -20 and $-30 \mathrm{~dB}[2]$. 
(a)

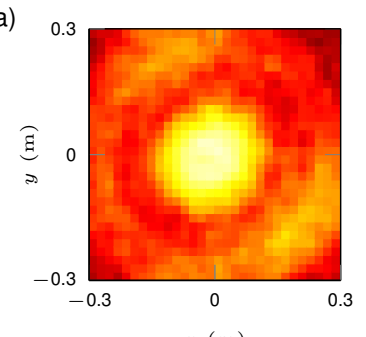

(d)

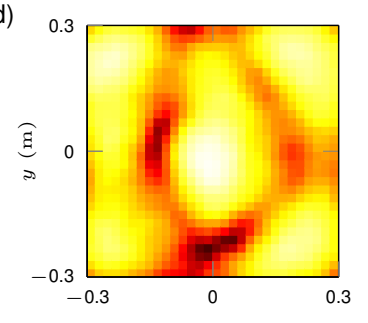

(g)

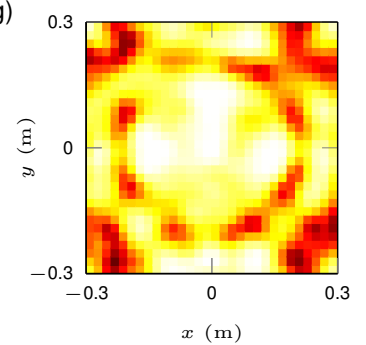

(b)

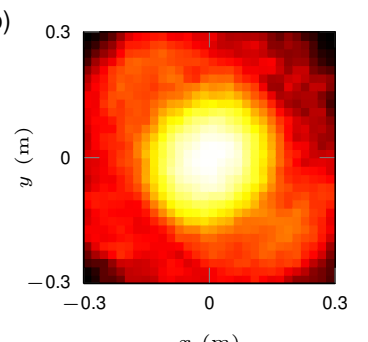

(e)

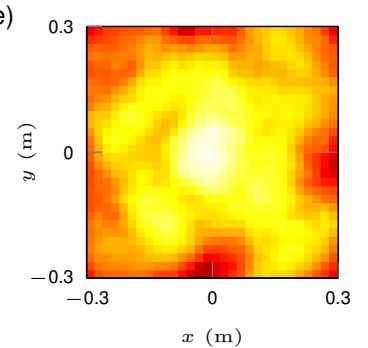

(h)

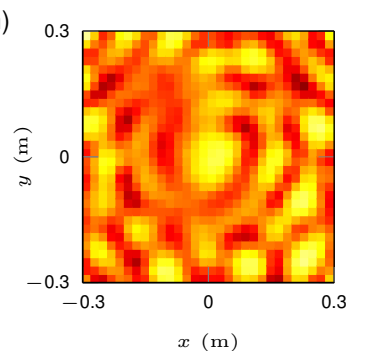

(c)

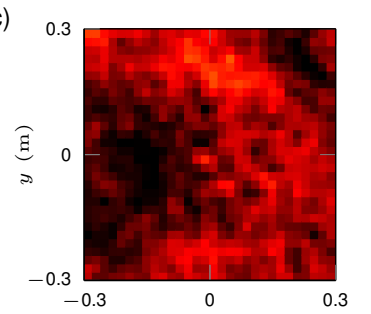

$x(\mathrm{~m})$

(f)

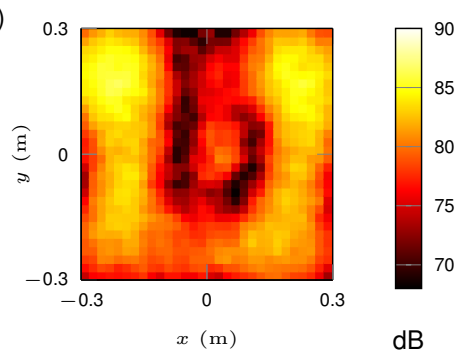

(i)

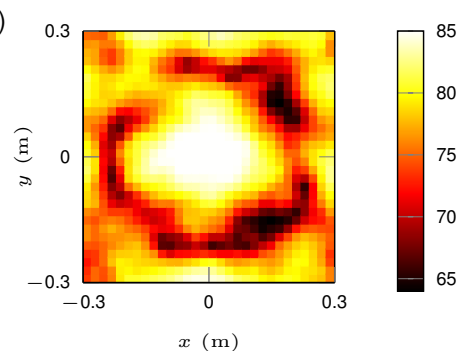

dB

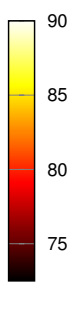

$\mathrm{dB}$

85

Figure 4: Sound pressure levels in $\mathrm{dB}$ re $20 \mu \mathrm{Pa}$ of the measured sound fields at $350 \mathrm{~Hz}$ (top row), $800 \mathrm{~Hz}$ (middle row) and $1800 \mathrm{~Hz}$ (bottom row). Left column: total sound field $\mathbf{p}_{h}$ (in the presence of the reflector). Middle column: direct sound field $\mathbf{p}_{h, \mathrm{ff}}$ (in the absence of the reflector). Right column: estimate of reverberant sound field $\mathbf{p}_{h, \mathrm{rv}}=\mathbf{p}_{h}-\mathbf{p}_{h, \mathrm{ff}}$.

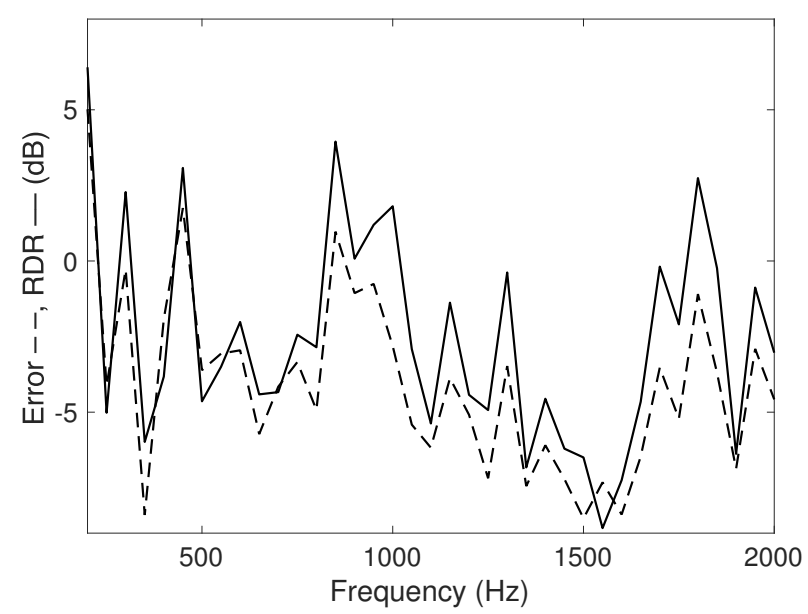

Figure 5: Dashed: reconstruction error of the free-field NAH method. Solid: reverberant-to-direct ratio (RDR).

However, when compared against the performance of double layer methods, such as that introduced by $\mathrm{Hu}$ et al. [21], it can be claimed that the reconstruction errors have similar orders of magnitude. This is 


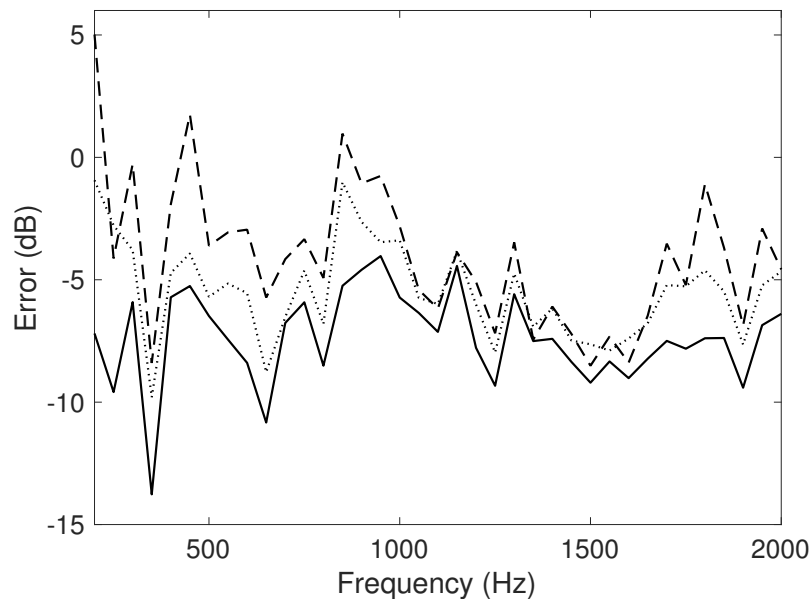

Figure 6: Dashed: reconstruction error of free-field NAH. Dotted: minimum reconstruction error of the first-order reflection (FOR) model via Eq. (17). Solid: minimum reconstruction error of the locally-reacting plate (LRP) model via Eq. (18).

partly due to the increased complexity of the acoustic problem to be solved, in contrast with a free-field measurement scenario. Moreover, the fact that only a single layer array is used makes the problem all the more challenging than for the case of double layer methods.

With the purpose of illustrating the minimum error approach into detail, Fig. 7 shows the reconstruction error via the FOR and LRP models at $1800 \mathrm{~Hz}$, projected onto the dimensions of $\beta_{1}$ and $\beta_{p}$. In spite of the FOR and LRP models having the same reflectivity function (recall Eq. (4)), it seems in Figs. 7(a) and (b) that the reflector admittance obtained with these models differ, and this can be attributed to the fact that $\beta_{1}$ obtained via the FOR model accounts for higher-order scattered and reflected fields, unlike the LRP model does. To be more exact, the admittance values that correspond to the minimum error are $\beta_{1}=0.5 \mathrm{j}$ and $0.02+0.07 \mathrm{j}$ for the FOR and LRP models respectively. On the other hand, the minimum in Fig. 7(c) gives a plate admittance of $-0.07 \mathrm{j}$ via the LRP model. The lack of convexity in these plots is at present attributed to the regularization parameter choice method adopted, which is the L-curve criterion and can sometimes have multiple local curvature maxima; however, this has not been encountered by the authors. A plot including the L-curves for all methods at $1800 \mathrm{~Hz}$ is shown in Fig. 8.

(a)

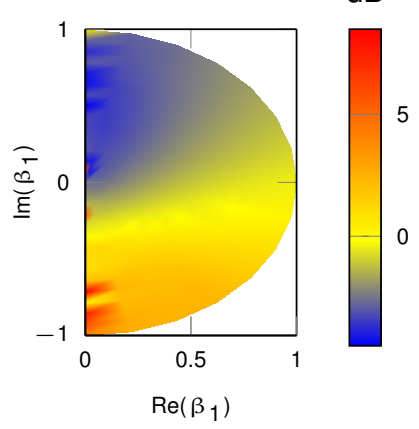

(b)

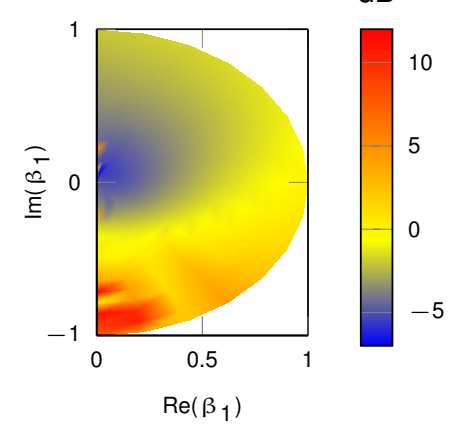

(c)

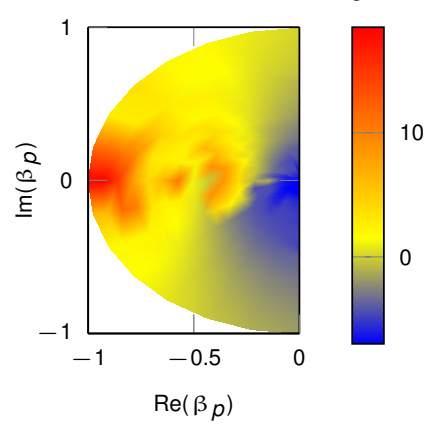

Figure 7: Illustration of the minimum error approach at $1800 \mathrm{~Hz}$ for the first-order reflection (FOR) and locally-reacting plate (LRP) models via Eqs. (17) and (18). (a) Projection of the reconstruction error, via the FOR model, onto the space of $\beta_{1}$. Projection of the reconstruction error, via the LRP model, onto the space of (b) $\beta_{1}$ and (c) $\beta_{p}$.

Fig. 9 below shows the reflector and plate admittances obtained via the minimum error approach over 


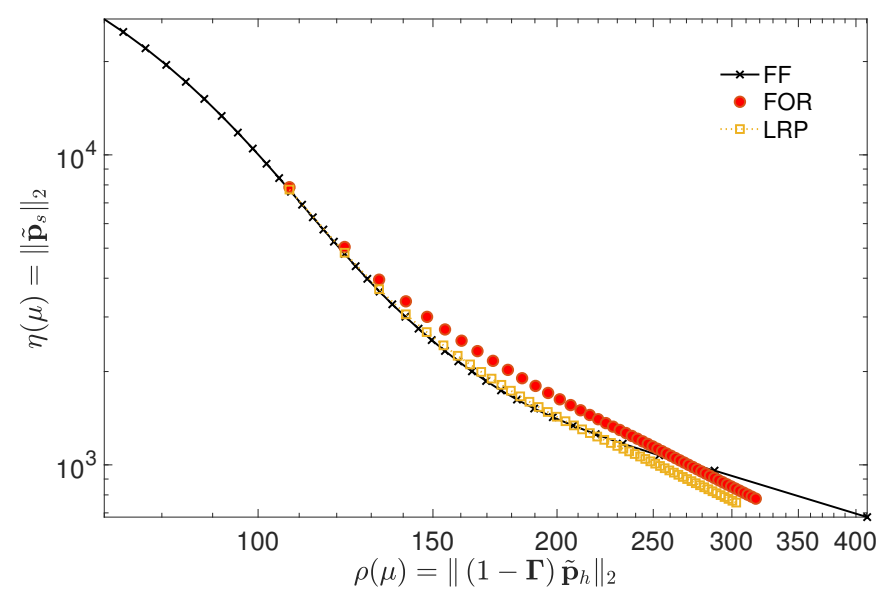

Figure 8: L-curves at $1800 \mathrm{~Hz}$ for the FF, FOR and LRP methods.

the whole frequency range. Fig. 9(a) and (c) show respectively the real and imaginary parts of the reflector admittance, obtained with the FOR and LRP models via Eqs. (17) and (18) respectively. Fig. 9(b) and (d) show the real and imaginary parts, respectively, of the plate admittance obtained with the LRP model via Eq. (18).

As seen in Figs. 9(a) and (c), there are certain similarities at some of the frequencies between the reflector admittances obtained with the FOR model and those obtained with the LRP model. This may be attributed to the RDR values at these frequencies: the lower the RDR, the lower the reverberant/scattered field. However, it appears that in general the reflector admittances obtained with these models differ quite substantially. As mentioned earlier, this can be due to the fact that, despite holding the same reflectivity response at $z_{1}$ as the LRP model, the minimum error approach with the FOR model accounts for the higher-order reflections by finding a potentially different reflector admittance $\beta_{1}$.

\subsubsection{Reconstructed sound fields with all methods}

Table 1 shows the reconstruction errors for all methods, as well as the RDR values at $350 \mathrm{~Hz}, 800 \mathrm{~Hz}$ and $1800 \mathrm{~Hz}$. Reconstructed SPLs with all methods at these frequencies are shown in Fig. 10. As anticipated in

Table 1: Reverberant-to-direct (RDR) ratio, and reconstruction errors obtained through the minimum error approach at 350 $\mathrm{Hz}, 800 \mathrm{~Hz}$ and $1800 \mathrm{~Hz}$, for the free-field (FF), first-order reflection (FOR), and locally-reacting plate (LRP) methods.

\begin{tabular}{lllll}
\hline \multirow{2}{*}{ Frequency $(\mathrm{Hz})$} & \multirow{2}{*}{ RDR $(\mathrm{dB})$} & \multicolumn{3}{c}{ Reconstruction error $(\mathrm{dB})$} \\
\cline { 3 - 5 } & & FF $[2]$ & FOR [5] & LRP \\
\hline 350 & -6 & -8.4 & -9.8 & -13.8 \\
800 & -2.9 & -4.9 & -6.8 & -8.5 \\
1800 & +2.7 & -1.1 & -4.6 & -7.4 \\
\hline
\end{tabular}

Section 3.2.2, Figs. 10(b), (f) and (j) indicate that the FF method performs better at $350 \mathrm{~Hz}$ than at $800 \mathrm{~Hz}$ and $1800 \mathrm{~Hz}$, and this is so because the RDR is lower at the former frequency. The reconstruction errors at these frequencies are respectively $-8.4 \mathrm{~dB},-4.9 \mathrm{~dB}$ and $-1.1 \mathrm{~dB}$. Comparison of Figs. 10(e) and (f), as well as (i) and (j), indicates that the reconstructed SPL via the FF method over-estimates the reference SPL, particularly at the center of the images. A possible explanation is that the excitation force is applied in the center of the plate, thus the reconstruction via the FF method is likely most contaminated by reflection and scattering in the neighborhood of the excitation point.

The reconstructions via the FOR model, shown in the middle-right column of Fig. 10, demonstrate a small improvement over the FF method. This is most noticeable at $800 \mathrm{~Hz}$ and $1800 \mathrm{~Hz}$, namely, by 
(a)

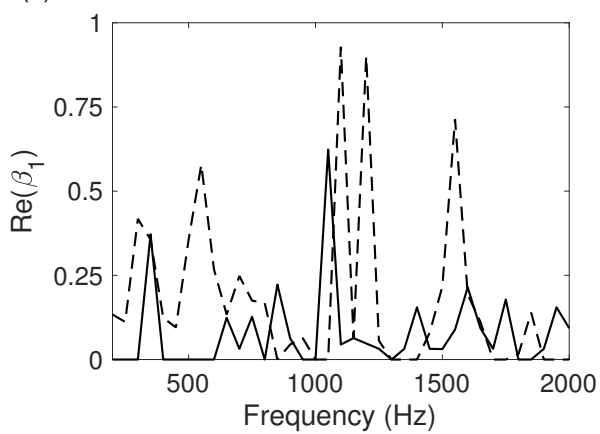

(c)

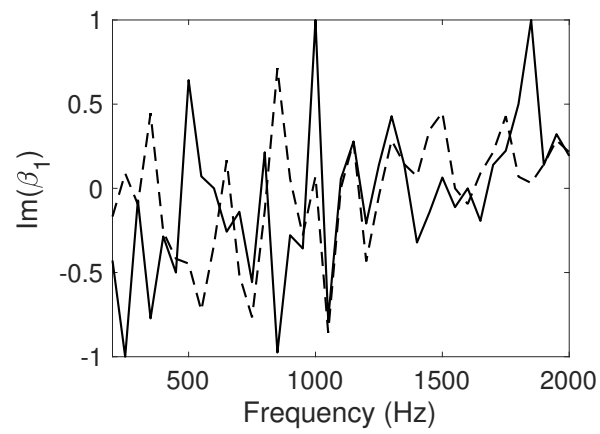

(b)

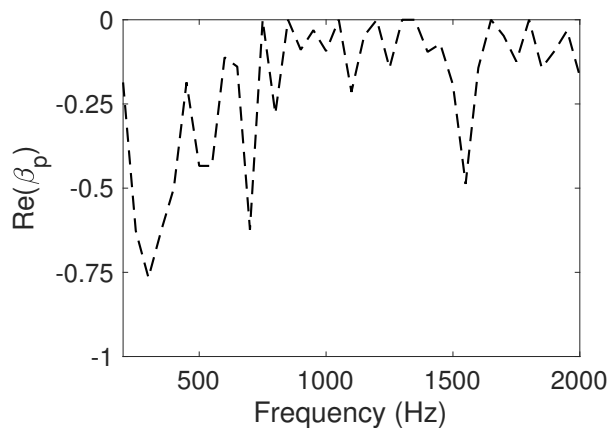

(d)

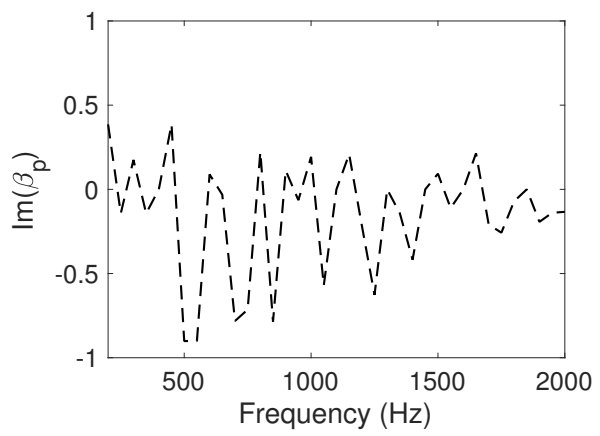

Figure 9: Optimal admittances of the reflector and the vibrating plate obtained through the minimum error approach. (a) Real and (c) imaginary part of the reflector admittance $\beta_{1}$ via FOR (solid) and LRP (dashed) models. (b) Real and (d) imaginary part of the plate admittance $\beta_{p}$ via the LRP model.

comparing Figs. 10(f) and (g), as well as (j) and (k). Moreover, by comparing Figs. 10(i) and (k), the reconstructed SPL via the FOR model over-estimates the reference SPL at the center of the image, similarly to the FF method. The reconstruction errors at the three frequencies are $-9.8 \mathrm{~dB},-6.8 \mathrm{~dB}$ and $-4.6 \mathrm{~dB}$ respectively.

As shown in the right-most column of Fig. 10, the LRP model appears to qualitatively perform best of all methods. The reconstruction errors at these frequencies are respectively $-13.8 \mathrm{~dB},-8.5 \mathrm{~dB}$ and -7.4 $\mathrm{dB}$. At these three frequencies, the reconstructions via the LRP model demonstrate an improvement over the FF and FOR methods, which can also be quantitatively confirmed in Figs. 5 and 7.

\section{Discussion}

\subsection{Cross-validation of admittance values}

In this section, a cross-validation of the admittance values is performed in order to investigate the robustness of the LRP model against different reconstruction planes. In few words, the pressure shall now be reconstructed at a plane $z_{s}=4 \mathrm{~cm}$ above the plate, and the estimated optimal admittances shall be compared with those in Fig. 9. The reflector and plate admittances versus frequency obtained with the LRP model at both reconstruction planes are then shown in Fig. 11.

It can at first be seen in Figs. 11(a) and (c) that the LRP reflector admittances at the two reconstruction planes have a few discrepancies around $500 \mathrm{~Hz}$ and in the frequency range between $1000 \mathrm{~Hz}$ and $1200 \mathrm{~Hz}$. In the remainder of the frequency range there appears to be very good agreement. As regards the plate admittances, depicted in Figs. 11(b) and (d), there is also good agreement between the two solutions. The largest differences occur in the real part at $1000 \mathrm{~Hz}$, as seen in Fig. 11(b), and in the imaginary part at $500 \mathrm{~Hz}$, as seen in Fig. 11(d). Overall, the results and analyses from the cross-validation presented in this 

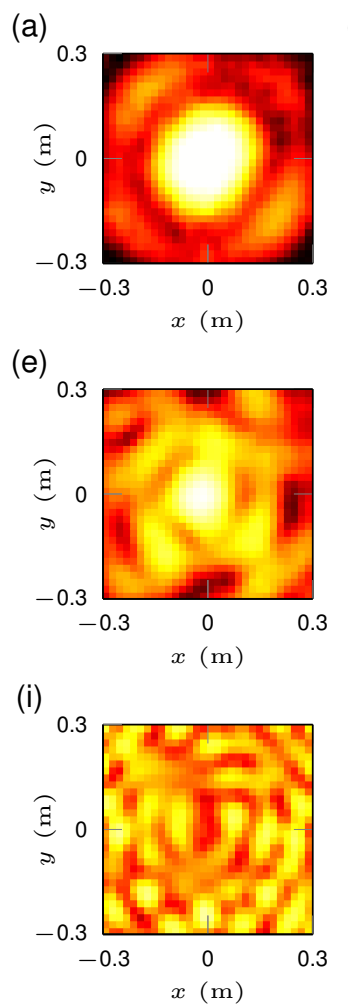

(b)

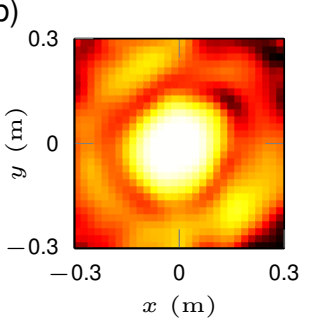

(f)

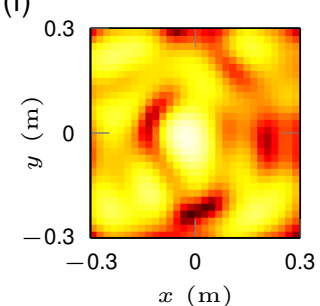

(j)

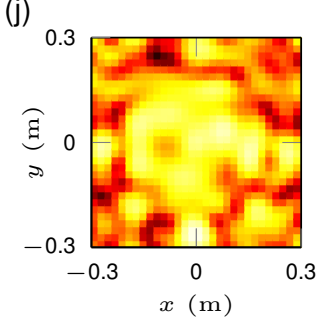

(c)

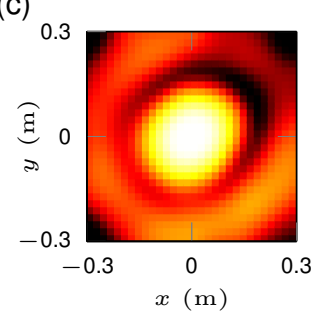

(g)

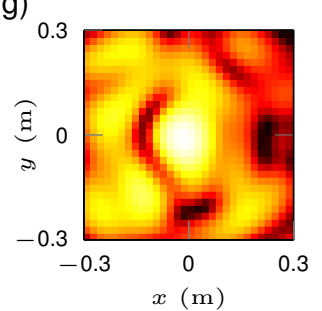

(k)

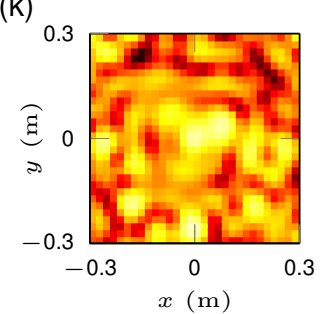

(d)

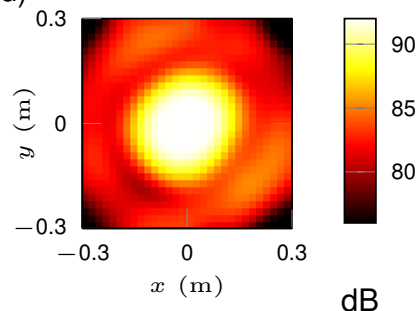

(h)

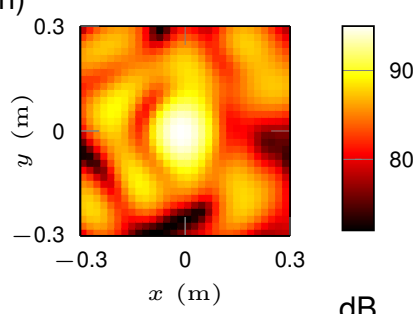

(l)

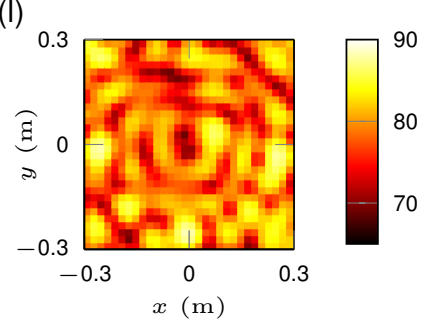

Figure 10: Reconstructed sound pressure levels in dB re $20 \mu \mathrm{Pa}$. Top row: $350 \mathrm{~Hz}$. Middle row: $800 \mathrm{~Hz}$. Bottom row: 1800 Hz. Left-most column: reference pressure. Middle-left column: reconstruction via the free-field method (FF). Middle-right column: reconstruction via the first-order reflection method (FOR) and Eq. (17). Right-most column: reconstruction via the locally-reacting plate method (LRP) and Eq. (18).

section indicate that, provided the admittances are known, there is potential for accurate reconstructions without knowledge of the reference pressure.

\subsection{On the applicability in practice}

It is also reasonable to question the applicability of the LRP method in case the reference source pressure is not available. On the one hand, the optimization functional in Eq. (11) is per se nonlinear due to the multiplicative nature of the admittances with the reconstructed source pressure. On the other hand, the problem is under-determined as there are only $M$ measurement points (equations) and $M+2$ unknowns. Although not investigated in this paper, one possible approach could be to employ, for instance, inexact Newton methods for under-determined problems. (For more details the interested reader is referred to the research by Simonis [37] as well as the references therein.) Other approaches, which may be more practical, involve the use of so-called impedance estimation methods. These can range from the well-known Tamura's technique to determine the plane-wave reflection coefficient with a linear microphone array [23, 24], to more recent techniques such as sparse array processing with spherical microphone arrays [38]. The general idea is to estimate the impedance of a material sample provided a known sound source (usually of monopole or dipole nature), and formulate a model that describes the acoustic propagation and reflection from the source to the array. This type of approach offers the advantage that the estimated impedance is angle-dependent, thus it is compatible with the reflectivity response in Eq. (4). 
(a)

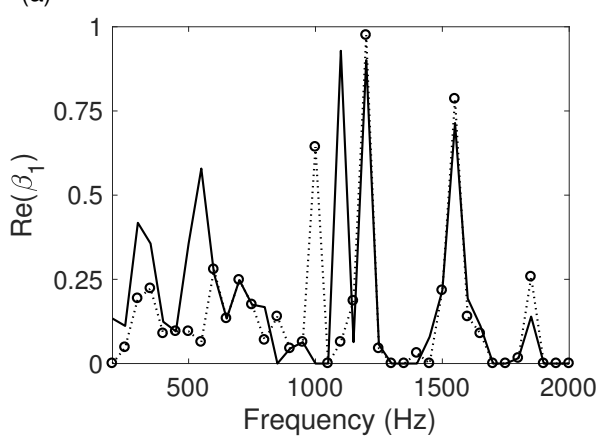

(c)

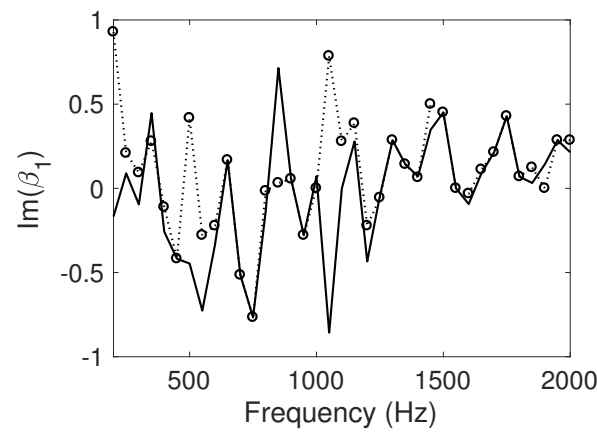

(b)

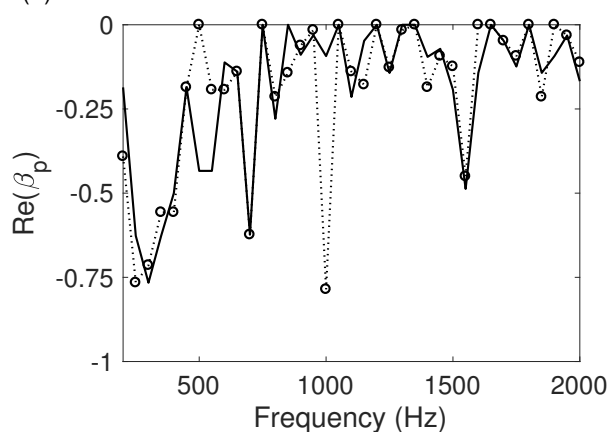

(d)

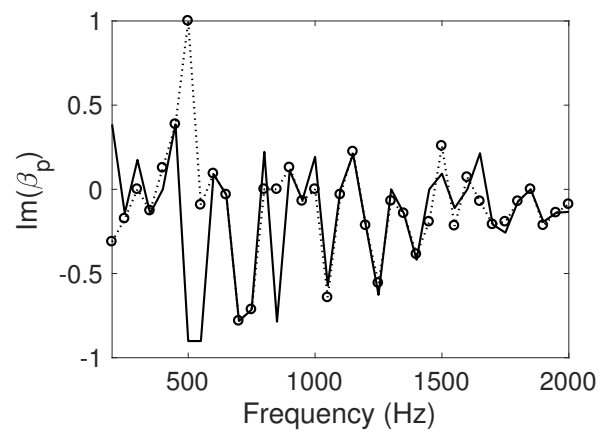

Figure 11: Optimal reflector and plate admittances obtained by computing the minimum error at two different reconstruction planes via the locally-reacting plate (LRP) model. Solid: $z_{s}=3 \mathrm{~cm}$ above the plate. Dotted: $z_{s}=4 \mathrm{~cm}$ above the plate. (a) Real and (c) imaginary part of the reflector admittance $\beta_{1}$. (b) Real and (d) imaginary part of the plate admittance $\beta_{p}$.

\subsection{Final remarks}

One of the consequences of the results in Fig. 11 is that the LRP method has the potential to estimate the reflector and plate admittances, provided the sound radiated by the plate in free-field is known and given as input. Additionally, as experimentally shown with the double layer array method proposed by Hu et al. [21], particle velocity reconstructions are more accurate than those of acoustic pressure. It remains thus the question whether this is also the case for the method introduced in this paper. Along similar lines, the formulation of the LRP model can in principle be easily modified in order to: (i) use a single layer array of particle velocity transducers instead of microphones, and (ii) further recover the plate normal velocity.

Lastly, it shall be mentioned that in practice the array aperture ought to be carefully chosen with respect to the dimensions of the source and the reflector. In other words, it is desirable to have a large enough array such that windowing artifacts are minimized and the wavenumber-domain resolution is sufficiently fine. However, for the source and the reflector most likely have finite dimensions, a too large of an array is likely to capture edge scattering effects and, as a consequence, provide unreliable sound field reconstructions.

\section{Summary}

The accuracy of planar near-field acoustic holography (NAH) is substantially dependent on the environment in which the measurement is performed. Today's solutions against this dependency are to move the source to an echo-free chamber, or to record and process the array data in two planes (or layers) so as to filter out the reflections. It is nevertheless not possible with a single layer array to separate and reconstruct the sound field radiated by a planar source that faces a parallel reflector. To this end, the present manuscript introduces and examines a single layer microphone array method, suited for measurements that are taken between a vibrating plate and a parallel reflecting surface. The technique, here referred to as locally-reacting 
plate (LRP) method, accounts for infinite reflected and scattered waves between the reflector and the plate, and the solution is formulated in closed-form in the wavenumber domain with the use of spatial Fourier transforms. As its name suggests, the LRP method assumes that the vibrating plate back-scatters sound in a locally-reacting manner. On the other hand, knowledge of the reflector and plate admittances is required if accurate reconstructions are aimed at. The method is tested with experiments and the reconstruction performance is compared against that of NAH in free-field conditions, as well as that of the so-called first-order reflection method, which neglects plate scattering and higher-order reflections. On the whole, it is shown with experiments that the introduced method can recover the free-field sound radiated by the plate with a reasonable accuracy.

\section{Acknowledgments}

The authors would like to acknowledge the anonymous reviewers for their valuable comments and suggestions. The first author would like to acknowledge the technical assistance with the experimental setup of Danilo Prelevic, Jens Sjölander and Luck Peerlings, affiliated to the Department of Aeronautical and Vehicle Engineering, KTH. This research is financially supported by the Swedish Research Council (Vetenskapsrådet) under grant numbers 2012-3723 and 2016-04366.

\section{Appendix A. Structure of the WRW matrices}

The WRW model defines the contents of the matrices $\mathbf{W}_{m n}$ as follows. Take the example of $\mathbf{W}_{s h}$, which relates the pressure propagation from $z=z_{s}$ to $z_{h}$. Then, the $n$-th column of $\mathbf{W}_{s h}$ represents the two-dimensional pressure field (impulse response) at $z=z_{h}$ due to a unit dipole (Dirac delta) at the spatial position $\left(x_{n}, y_{n}, z_{s}\right)$. In mathematical terms, the $n$-th column of $\mathbf{W}_{s h}$ can be defined as [26]

$$
\left\{\mathbf{W}_{s h}\right\}_{n}=\left(z_{h}-z_{s}\right)(1+\mathrm{j} k \Delta r) \frac{e^{-\mathrm{j} k \Delta r}}{2 \pi \Delta r^{3}} \Delta x \Delta y
$$

where $\Delta x$ and $\Delta y$ is the sensor spacing in the $x$ and $y$ coordinates, and

$$
\Delta r=\left[\left(x-x_{n}\right)^{2}+\left(y-y_{n}\right)^{2}+\left(z_{h}-z_{s}\right)^{2}\right]^{1 / 2} .
$$

On the other hand, the reflectivity matrices $\mathbf{R}_{i}$ are diagonal when the associated surface $z=z_{i}$ is deemed locally-reacting $[39,26]$, and the diagonal elements correspond with the reflection coefficients at the points in the plane.

\section{Appendix B. Wavenumber-domain equivalence with the WRW model for two reflectors}

In this appendix it is shown that the derivation presented in Eq. (9) for the locally-reacting plate is equivalent to the wavenumber-domain equivalent of the WRW model for two parallel reflectors [26], assuming that the plate is one of these reflectors. We shall depart from the main result of the WRW model, namely the equation

$$
\mathbf{p}_{h}=\left[\mathbf{W}_{s h}+\sum_{q=1}^{\infty} \mathbf{W}_{h}(\mathbf{R W})^{q-1} \mathbf{R} \mathbf{W}_{s}\right] \mathbf{p}_{s},
$$

where $\mathbf{p}_{s}$ is the pressure field at a plane $z=z_{s}$ parallel to the array, such that $z_{p}<z_{s}<z_{h}$. This pressure field is related to the direct (free-field) sound field in Eq. (1) via $\mathbf{p}_{h, \mathrm{ff}}=\mathbf{W}_{s h} \mathbf{p}_{s}$. In addition, the following expressions hold

$$
\begin{aligned}
\mathbf{W}_{h} & =\left(\begin{array}{ll}
\mathbf{W}_{h p} & \mathbf{W}_{h 1}
\end{array}\right), \\
\mathbf{R} & =\left(\begin{array}{cc}
\mathbf{R}_{p} & \mathbf{0} \\
\mathbf{0} & \mathbf{R}_{1}
\end{array}\right),
\end{aligned}
$$




$$
\begin{aligned}
& \mathbf{W}=\left(\begin{array}{cc}
\mathbf{0} & \mathbf{W}_{p 1} \\
\mathbf{W}_{1 p} & \mathbf{0}
\end{array}\right), \\
& \mathbf{W}_{s}=\left(\begin{array}{ll}
\mathbf{0} & \mathbf{W}_{s 1}
\end{array}\right)^{T},
\end{aligned}
$$

where superscript $T$ denotes matrix transpose.

Because of the convolution theorem as well as the linearity property of the spatial Fourier transform, Eq. (B.1) can be expressed in the wavenumber domain as

$$
\tilde{\mathbf{p}}_{h}=\left[\mathbf{G}_{s h}+\sum_{q=1}^{\infty}\left(\begin{array}{ll}
\mathbf{G}_{p h} & \mathbf{G}_{h 1}
\end{array}\right)\left(\begin{array}{cc}
\mathbf{0} & \tilde{\mathbf{R}}_{p} \mathbf{G}_{p 1} \\
\tilde{\mathbf{R}}_{1} \mathbf{G}_{p 1} & \mathbf{0}
\end{array}\right)^{q-1}\left(\begin{array}{c}
\mathbf{0} \\
\tilde{\mathbf{R}}_{1} \mathbf{G}_{s 1}
\end{array}\right)\right] \tilde{\mathbf{p}}_{s},
$$

where the sum gives a Neumann series that leads to the result

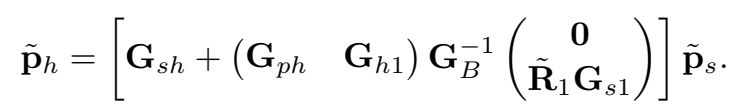

Here it follows that

$$
\mathbf{G}_{B}=\left(\begin{array}{cc}
\mathbf{I} & -\tilde{\mathbf{R}}_{p} \mathbf{G}_{p 1} \\
-\tilde{\mathbf{R}}_{1} \mathbf{G}_{p 1} & \mathbf{I}
\end{array}\right) .
$$

Since the latter operator is a block matrix, we can find the inverse by calculating its Schur complements. From Schur's formula, the inverse of $\mathbf{G}_{B}$ exists if one of the main diagonal blocks, and its corresponding Schur complement, is invertible [40]. It is trivial that the main blocks are invertible, thus we proceed to examine the Schur complement, which is defined as

$$
\mathbf{\Phi}=\mathbf{I}-\tilde{\mathbf{R}}_{p} \tilde{\mathbf{R}}_{1} \mathbf{G}_{p 1}^{2} .
$$

Only when the admittances of the reflector and the plate are both exactly zero, i.e. acoustically rigid, the Schur complement is non-invertible. In that case, the singularity condition can be obtained by equating $\boldsymbol{\Phi}$ to zero, which gives the relationship

$$
k^{2}=k_{x}^{2}+k_{y}^{2}+\xi^{2} \frac{\pi^{2}}{\left(z_{1}-z_{p}\right)^{2}}, \quad \xi \in \mathbb{N} .
$$

Despite the theoretical admissibility of this case, it is quite unlikely in practice that both the reflector and the plate are acoustically rigid; hence we could expect that $\mathbf{\Phi}$ is well conditioned for most practical situations. Then the inverse reads

$$
\mathbf{G}_{B}^{-1}=\left(\begin{array}{cc}
\boldsymbol{\Phi}^{-1} & \tilde{\mathbf{R}}_{p} \mathbf{G}_{p 1} \boldsymbol{\Phi}^{-1} \\
\tilde{\mathbf{R}}_{1} \mathbf{G}_{p 1} \boldsymbol{\Phi}^{-1} & \boldsymbol{\Phi}^{-1}
\end{array}\right)
$$

and the total sound field is defined as

$$
\tilde{\mathbf{p}}_{h}=\left[\mathbf{G}_{s h}+\left(\mathbf{G}_{h 1}+\mathbf{G}_{p h} \tilde{\mathbf{R}}_{p} \mathbf{G}_{p 1}\right) \boldsymbol{\Phi}^{-1} \tilde{\mathbf{R}}_{1} \mathbf{G}_{s 1}\right] \tilde{\mathbf{p}}_{s} .
$$

Lastly, Eq. (9) can be recovered by using the relationship $\tilde{\mathbf{p}}_{h, \mathrm{ff}}=\mathbf{G}_{s h} \tilde{\mathbf{p}}_{s}$ in the above equation.

\section{Appendix C. Singularities of G}

The singularity condition for $\mathbf{G}$ follows from equating Eq. (9) to zero, which leads to

$$
\left(k_{z}-k \beta_{1}\right) \mathrm{e}^{2 \mathrm{j} k_{z}\left(z_{1}-z_{h}\right)}+k_{z}+k \beta_{1}=0 .
$$

By making a power series expansion of the exponential term, the following polynomial approximation is obtained

$$
\left(k_{z}-k \beta_{1}\right)\left(1+\sum_{u=1}^{U} \varsigma_{u} k_{z}^{u}\right)+k_{z}+k \beta_{1} \approx 0
$$


with $\varsigma_{u}=\left[2 \mathrm{j}\left(z_{1}-z_{h}\right)\right]^{u} / u$ ! and $U \gg 1$. As shown in [5], the $U+1$ polynomial roots of $k_{z}$ consist of $U$ complex roots and one real root. This factorization is possible for the polynomial begins with first-order terms of $k_{z}$. In addition, a complex $k_{z}$ root is inadmissible because the acoustic medium is not deemed viscous. Thus, the spectrum operator $\mathbf{G}$ is singular at the radiation circle, that is, when $k^{2}=k_{x}^{2}+k_{y}^{2}$.

To put this in a practical context, the discretization of the wavenumber domain is defined by $k_{x}=$ $2 \pi m / L_{x}$ and $k_{y}=2 \pi n / L_{y}$, where $L_{x}$ and $L_{y}$ are the array lengths along the $x$ and $y$ coordinates, and $m$ and $n$ are integers. Then, if the array length is equal along the two spatial coordinates, i.e. $L_{x}=L_{y}=L$, the singularity will be determined by

$$
L=\lambda \sqrt{m^{2}+n^{2}} .
$$

This type of singularity is not new, and the mathematics have appeared in several other occasions, such as the wavenumber-domain computation of the Rayleigh integral for planar sources [41].

\section{References}

[1] J. D. Maynard, E. G. Williams, Y. Lee, Nearfield acoustic holography: I. Theory of generalized holography and the development of NAH, The Journal of the Acoustical Society of America 78 (4) (1985) 1395-1413. doi:10.1121/1.392911. URL http://asa.scitation.org/doi/10.1121/1.392911

[2] E. G. Williams, Fourier Acoustics: Sound Radiation and Nearfield Acoustical Holography, Academic Press, San Diego, 1999.

[3] J. Pachner, Investigation of scalar wave fields by means of instantaneous directivity patterns, The Journal of the Acoustical Society of America 28 (1) (1956) 90-92. doi:10.1121/1.1908234.

[4] G. Weinreich, E. B. Arnold, Method for measuring acoustic radiation fields, The Journal of the Acoustical Society of America 68 (2) (1980) 404-411. doi:10.1121/1.384751.

[5] E. Zea, I. Lopez Arteaga, Single layer planar near-field acoustic holography for compact sources and a parallel reflector, Journal of Sound and Vibration 380 (2016) 129-145. doi:10.1016/j.jsv.2016.06.012. URL http://linkinghub.elsevier.com/retrieve/pii/S0022460X16302346

[6] C.-X. Bi, X.-Z. Chen, J. Chen, Sound field separation technique based on equivalent source method and its application in nearfield acoustic holography., The Journal of the Acoustical Society of America 123 (3) (2008) 1472-1478. doi:10.1121/1.2837489.

[7] Y.-B. Zhang, F. Jacobsen, C.-X. Bi, X.-Z. Chen, Near field acoustic holography based on the equivalent source method and pressure-velocity transducers., The Journal of the Acoustical Society of America 126 (3) (2009) 1257-1263. doi:10.1121/1.3179665.

[8] E. Fernandez-Grande, F. Jacobsen, Q. Leclère, Sound field separation with sound pressure and particle velocity measurements., The Journal of the Acoustical Society of America 132 (6) (2012) 3818-3825. doi:10.1121/1.4763988.

[9] C.-X. Bi, D.-Y. Hu, Y.-B. Zhang, J. S. Bolton, Reconstruction of the free-field radiation from a vibrating structure based on measurements in a noisy environment, The Journal of the Acoustical Society of America 134 (4) (2013) $2823-2832$.

[10] C.-X. Bi, W.-Q. Jing, Y.-B. Zhang, L. Xu, Patch nearfield acoustic holography combined with sound field separation technique applied to a non-free field, Science China Physics, Mechanics \& Astronomy 58 (2) (2015) 1-9.

[11] C.-X. Bi, D.-Y. Hu, Y.-B. Zhang, W.-Q. Jing, Identification of active sources inside cavities using the equivalent source method-based free-field recovery technique, Journal of Sound and Vibration 346 (2015) 153-164.

[12] J. Hald, Patch holography in cabin environments using a two-layer handheld array with an extended SONAH algorithm, in: Proc. EuroNoise, Tampere, 2006

[13] F. Jacobsen, V. Jaud, Statistically optimized near field acoustic holography using an array of pressure-velocity probes., The Journal of the Acoustical Society of America 121 (3) (2007) 1550-1558. doi:10.1121/1.2434245.

[14] F. Jacobsen, X. Chen, V. Jaud, A comparison of statistically optimized near field acoustic holography using single layer pressure-velocity measurements and using double layer pressure measurements., The Journal of the Acoustical Society of America 123 (4) (2008) 1842-1845. doi:10.1121/1.2875308.

[15] Y.-B. Zhang, X.-Z. Chen, F. Jacobsen, A sound field separation technique based on measurements with pressure-velocity probes (L). The Journal of the Acoustical Society of America 125 (6) (2009) 3518-3521. doi:10.1121/1.3127128.

[16] E. Fernandez-Grande, F. Jacobsen, Sound field separation with a double layer velocity transducer array (L)., The Journal of the Acoustical Society of America 130 (1) (2011) 5-8. doi:10.1121/1.3598431.

[17] J. H. Deng, J. H. Dong, G. D. Meng, Sound source identification and acoustic contribution analysis using nearfield acoustic holography, Advanced Materials Research 945-949 (2014) 717-724.

[18] M. Villot, G. Chavériat, J. Roland, Phonoscopy: An acoustical holography technique for plane structures radiating in enclosed spaces, The Journal of the Acoustical Society of America 91 (1) (1992) 187-195. doi:10.1121/1.402766.

[19] D. Hallman, J. S. Bolton, A technique for performing source identification in a reflective environment by using nearfield acoustical holography, in: Proc. Noise-Con, Williamsburg, 1993, pp. 479-484.

[20] E. G. Williams, The nearfield acoustical holography (NAH) experimental method applied to vibration and radiation in light and heavy fluids, Computers \& Structures 65 (3) (1997) 323-335. doi:10.1016/S0045-7949(96)00253-2.

[21] D.-Y. Hu, C.-X. Bi, Y.-B. Zhang, L. Geng, Extension of planar nearfield acoustic holography for sound source identification in a noisy environment, Journal of Sound and Vibration 333 (24) (2014) 6395-6404. doi:10.1016/j.jsv.2014.07.015. 
[22] G. V. Frisk, A. V. Oppenheim, D. R. Martinez, A technique for measuring the plane-wave reflection coefficient of the ocean bottom, The Journal of the Acoustical Society of America 68 (2) (1980) 602-612. doi:10.1121/1.384772.

[23] M. Tamura, Spatial Fourier transform method of measuring reflection coefficients at oblique incidence. I: Theory and numerical examples, The Journal of the Acoustical Society of America 88 (5) (1990) 2259-2264. doi:10.1121/1.400068.

[24] M. Tamura, J. F. Allard, D. Lafarge, Spatial Fourier-transform method for measuring reflection coefficients at oblique incidence. II. Experimental results, The Journal of the Acoustical Society of America 97 (4) (1995) $2255-2262$.

[25] A. J. Berkhout, A unified approach to acoustical reflection imaging. I: The forward model, The Journal of the Acoustical Society of America 93 (4) (1993) 2005-2016. doi:10.1121/1.406714.

[26] A. J. Berkhout, D. de Vries, J. Baan, B. W. van den Oetelaar, A wave field extrapolation approach to acoustical modeling in enclosed spaces, The Journal of the Acoustical Society of America 105 (3) (1999) 1725-1733. doi:10.1121/1.426710.

[27] P. M. Morse, K. U. Ingard, Theoretical Acoustics, McGraw-Hill, New York, 1968. doi:10.1119/1.1976432.

[28] J. W. Goodman, Introduction to Fourier Optics, 2nd Edition, McGraw-Hill, London, 1996.

[29] P. Brémaud, Mathematical Principles of Signal Processing - Fourier and Wavelet Analysis, Springer, New York, 2002.

[30] G. V. Frisk, Inhomogeneous waves and the plane-wave reflection coefficient, The Journal of the Acoustical Society of America 66 (1) (1979) 219-234. doi:10.1121/1.383074.

[31] E. G. Williams, Regularization methods for near-field acoustical holography, The Journal of the Acoustical Society of America 110 (4) (2001) 1976-1988. doi:10.1121/1.1404381.

[32] R. Scholte, I. Lopez, N. B. Roozen, H. Nijmeijer, Wavenumber domain regularization for near-field acoustic holography by means of modified filter functions and cut-off and slope iteration, Acta Acustica united with Acustica 94 (3) (2008) 339-348. doi:10.3813/AAA.918041.

[33] P. C. Hansen, D. P. O'Leary, The use of the L-curve in the regularization of discrete ill-posed problems, SIAM Journal on Scientific Computing 14 (6) (1993) 1487-1503.

[34] G. H. Golub, M. Heath, G. Wahba, Generalized cross-validation as a method for choosing a good ridge parameter, Technometrics 21 (2) (1979) 215-223.

[35] V. A. Morozov, On the solution of functional equations by the method of regularization, Soviet Math. Dokl. 7 (1966) 414-417.

[36] I. Lopez Arteaga, R. Scholte, H. Nijmeijer, Improved source reconstruction in Fourier-based Near-field Acoustic Holography applied to small apertures, Mechanical Systems and Signal Processing 32 (2012) 359-373. doi:10.1016/j.ymssp.2012.06.002. URL http://linkinghub.elsevier.com/retrieve/pii/S0888327012002373

[37] J. P. Simonis, Inexact Newton Methods Applied to Under-Determined Systems, Phd thesis, Worcester Polytechnic Institute (2006).

URL https://www.wpi.edu/Pubs/ETD/Available/etd-050406-103442/unrestricted/simonis.pdf

[38] A. Richard, E. Fernandez-Grande, J. Brunskog, C.-H. Jeong, Estimation of surface impedance at oblique incidence based on sparse array processing, The Journal of the Acoustical Society of America 141 (6) (2017) 4115-4125. doi:10.1121/1.4983756. URL http://asa.scitation.org/doi/10.1121/1.4983756

[39] C. G. M. de Bruin, C. P. A. Wapenaar, A. J. Berkhout, Angle-dependent reflectivity by means of prestack migration, Geophysics 55 (9) (1990) 1223-1234. doi:10.1190/1.1442938.

[40] F. Zhang, The Schur Complement and its Applications, Springer, New York, 2005.

[41] E. G. Williams, J. D. Maynard, Numerical evaluation of the Rayleigh integral for planar radiators using the FFT, The Journal of the Acoustical Society of America 72 (6) (1982) 2020-2030. 